\title{
A Black-box Model for Neurons
}

\author{
N. Roqueiro \\ Federal University of Santa Catarina \\ Florianopolis (Brasil) \\ nestor.roqueiro@ufsc.br
}

\author{
C. Claumann \\ Researcher \\ Florianopolis (Brasil) \\ carlos.claumann@posgrad.ufsc.br
}

\author{
A. Guillamon, E. Fossas \\ Universitat Politècnica de Catalunya \\ Barcelona (Spain) \\ \{antoni.guillamon,enric.fossas\}@upc.edu
}

\begin{abstract}
We explore the identification of neuronal voltage traces by artificial neural networks based on wavelets (Wavenet). More precisely, we apply a modification in the representation of dynamical systems by Wavenet which decreases the number of used functions; this approach combines localized and global scope functions (unlike Wavenet, which uses localized functions only). As a proof-of-concept, we focus on the identification of voltage traces obtained by simulation of a paradigmatic neuron model, the Morris-Lecar model. We show that, after training our artificial network with biologically plausible input currents, the network is able to identify the neuron's behaviour with high accuracy, thus obtaining a black box that can be then used for predictive goals. Interestingly, the interval of input currents used for training, ranging from stimuli for which the neuron is quiescent to stimuli that elicit spikes, shows the ability of our network to identify abrupt changes in the bifurcation diagram, from almost linear input-output relationships to highly nonlinear ones. These findings open new avenues to investigate the identification of other neuron models and to provide heuristic models for real neurons by stimulating them in closed-loop experiments, that is, using the dynamic-clamp, a well-known electrophysiology technique.
\end{abstract}

\section{INTRODUCTION}

Neurons are the basic information processing structures in the brain. Through synapses or direct stimulation, neurons receive input signals that are shaped within the cell according to its intrinsic properties: morphology, ionic channels, availability of neurotransmitters,... The main observable of these transformations are voltage changes in the membrane potential (i.e., the difference in electrical potential between the interior and the exterior of a neuron). There is a vast literature on modeling of such intrinsic features, see for instance [3] for a thorough treatment. The main formalism was introduced by Hodgkin and Huxley [2] and consists of modeling the membrane potential of the neuron with the help of Kirchhoff's laws and first order kinetics describing the probability of specific ionic channels (sensitive to sodium, potassium, calcium or other chemical elements) to be open/closed. A plethora of experiments has since been devoted to provide specific models by identifying and quantifying the ionic channels, giving rise to very precise biophysical models now available to the computational neuroscience community. However, these are costly experiments that cannot be performed for every single trace obtained in electrophysiology labs, and so there exists a huge amount of experimental data not associated to a biophysically-derived mathematical model. Thus, the problem of identification and cell classification from voltage traces is fundamental to experimental neuroscience, see [5] where the problem of detection, time-estimation, and cell classification is treated in order to sort neural action potentials. Here, we use a computationally efficient modification of classical Wavelet-based networks to identify dynamics of cells from voltage traces. The identification method decreases the number of used functions in Wavenet by combining localized and global scope functions instead of only localized functions. In order to test the goodness of this estimation tool, we have chosen a benchmark neuron model, the Morris-Lecar model, a paradigmatic biophysical model able to reproduce the main states of a neuron, namely quiescent state and regular spiking, with only constant current stimuli. We train the modified wavenet using voltage traces obtained after applying a variable input current stimulus, that sweeps a biologically plausible interval, to the differential equations that define the MorrisLecar model. This procedure allows to identify the parameters with a best fit to the data and, ultimately, it provides a blackbox model of the neuron that can be used as a predictive or inference tool.

\section{NEURON MODEL}

As a benchmark neuron model, we consider the MorrisLecar model proposed in [1], which has been profusely used in computational neuroscience as it models fundamental types of neural dynamics while it is still feasible to make a qualitative analysis of it, see [4]. The dynamics of the neuron is modeled by a continuous-time dynamical system composed of the current-balance equation for the membrane potential, $v=v(t)$, and the $K^{+}$gating variable $0 \leq w=w(t) \leq 1$, which represents the probability of the $K^{+}$ionic channel to be active:

$$
\begin{aligned}
C_{m} \frac{d v}{d t} & =-g_{L}\left(v-E_{L}\right)-g_{K} w\left(v-E_{K}\right) \\
& -g_{C a} m_{\infty}(v)\left(v-E_{C a}\right)+I_{a p p}, \\
\frac{d w}{d t} & =\phi \frac{w_{\infty}(v)-w}{\tau_{w}(v)} .
\end{aligned}
$$

The leakage, calcium, and potassium currents are of the form $I_{L}=g_{L}\left(v-E_{L}\right), I_{C a}=g_{C a} m_{\infty}(v)\left(v-E_{C a}\right)$, and $I_{K}=g_{K} w\left(v-E_{K}\right)$, respectively; $g_{L}, g_{C a}$ and $g_{K}$ are the maximal conductances of each current, whereas $E_{L}, E_{C a}$ and $E_{K}$ denote the Nernst equilibrium potentials, for which the corresponding current is zero, also known as reversal potentials. The constant $C_{m}=20 \mu \mathrm{F} / \mathrm{cm}^{2}$ is the membrane capacitance, $\phi=1 / 15$ is a dimensionless constant, $I_{a p p}$ 


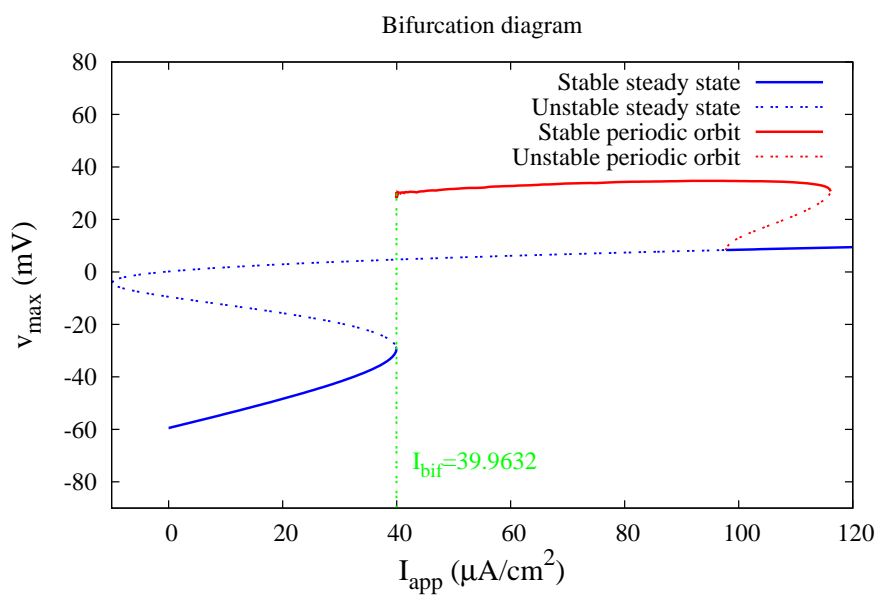

Fig. 1. Bifurcation diagram of system (1) in terms of the parameter $I_{a p p}$. The graph shows the maximal value of the variable $v$ on the equilibrium points or on the periodic orbits which, indeed, are limit cycles.

represents the (externally) applied current, which will be variable in our simulations, and

$$
\begin{aligned}
m_{\infty}(v) & =\left(1+\tanh \left(\left(v-V_{1}\right) / V_{2}\right)\right) / 2, \\
w_{\infty}(v) & =\left(1+\tanh \left(\left(v-V_{3}\right) / V_{4}\right)\right) / 2, \\
\tau_{w}(v) & =\left(\cosh \left(\left(v-V_{3}\right) /\left(2 V_{4}\right)\right)\right)^{-1},
\end{aligned}
$$

The following set of parameters have been used in our computations, see [4]:

$$
\begin{aligned}
& E_{L}=-60, E_{K}=-84, E_{C a}=120(\mathrm{mV}), \\
& V_{1}=-1.2, V_{2}=18, V_{3}=12, V_{4}=17.4(\mathrm{mV}), \\
& g_{L}=2, g_{K}=8.0, g_{C a}=4.0\left(\mathrm{mS} / \mathrm{cm}^{2}\right) .
\end{aligned}
$$

Figure II, shows the bifurcation diagram of system (1) in terms of the parameter $I_{a p p}$. In the experiments, a prescribed $I_{a p p}(t)$ that spans from 20 to $80 \mu \mathrm{A} / \mathrm{cm}^{2}$ was used. Note that for $I_{a p p}$ below $I_{b i f} \approx 39.9632$, there is one attractor, which is an equilibrium point of the system, while for $I_{a p p} \in\left(I_{b i f}, 80\right)$, also there is a unique attractor, which is a limit cycle (only the maximal value of the variable $v$ on the limit cycle is shown).

\section{THE NETWORK CHARACTERISTICS}

Great advances were made in the last years in analysis and identification of dynamical systems using non-linear models originated from artificial intelligence. In this area, models obtained from syntax rules (fuzzy logic) and, mainly, those ones that use activation functions (neural networks) are significative. In the artificial intelligence approach, a neural network comprises neurons layers, interconnected through weights. Mathematically, they are complex models, whose structure is empirically determined.

The most used neural network for control and non-linear system identification is the feedforward. Nearly $90 \%$ of the works found in the literature use this kind of network. A great part of this success can be attributed to the iterative algorithm used in the supervised training, known as Backpropagation [6]. Nevertheless, systems identification can be very tiring, due to the great number of network structure parameters (number of hidden layers, number of neurons per layer) and training method (weights, initial selection, learning factor determination, moment rate and stopping criteria) [7]. Network structure parameters and training method are determined by error and trial or Heuristic.

Due to the great number of parameters and lack of mathematical basis, feedforward networks have been replaced by non-linear models, but linear on its parameters. This structure is very attractive, because training can be formalized as a linear regression problem and, hence, solved by least squares. Two kinds of non-linear networks, linear on its parameters, have been used: radial base function networks (RBFN) and, most recently, wavelet networks. RBFN have only one hidden layer, whose neurons use activation functions, generally with compact support and defined around centers [7]. Network structure parameters are defined by number and center location. Compared to feedforward, RBFN needs a smaller number of parameters. Wavelet networks are made of localized functions, as RBFN. However, they are better mathematically based.

Wavelet networks use the multiresolution concept. [8]. Analysis in multiresolution is a structure of signal representation, in different scales or resolutions. A signal in the multiresolution frame is represented as the sum of successive approximations, done from projections of this signal in spaces defined in wavelets theory [9], [10].

The use of wavelets in approximations of functions and neural network construction came out with Bakshi [11], through the wavenets and Zhang [12] with frame networks. In the multiresolution frame, the approximation of a function $f(x)$ is made through its projections to shifted and compressed versions of a basic function, known as "wavelet mother". Translations and compressions and, thus, location and size are defined by wavelet theory. In this case, network training is restricted to determine the coefficients (weights) relative to projections. The issue is that the number of activation functions of a wavenet grows in an exponential way, as the number of inputs becomes larger. In addition, the activation functions support decreases too much, in relation to the problem domain, since in a wavenet the support of each multidimensional activation function is obtained from the intersection of the unidimensional localized function supports. Hence, there may be functions with a very small number of points in its support, causing numerical problems to training, mainly when there is deficient data sampling.

In [17], an approach to reduce the number of activation functions in a wavenet is proposed. Training data are initially approximated with activation functions (scale functions), whose support is equal to the problem domain support (global scope functions), different from the originally proposed wavenet, which uses localized functions only. If approximation is not adequate, thus wavelets with an increasing level of location could be added, according to multiresolution. 


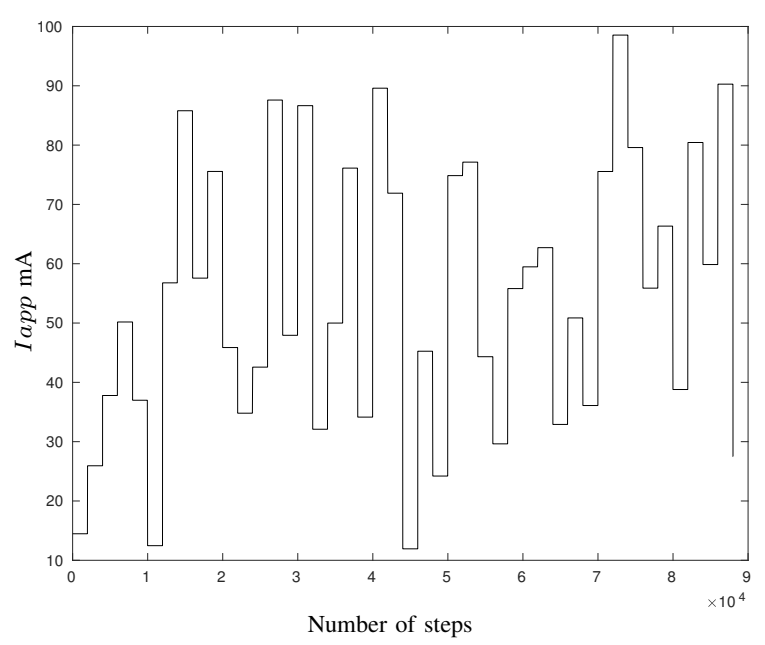

Fig. 2. Externally applied current

\section{THE WAVENET MODEL}

\section{A. Dynamical system identification}

In this work, we deal with the identification of the neuronal voltage traces of the Morris-Lecar model proposed in [1]. The steps followed in the identification process were:

1) Acquisition of data group for fitting (Training Patterns): data were obtained solving system 2 .

2) Determination of the best network structure: the set of input variables that better identifies the process has been selected in this step. As a measure criterion, the smaller quadratic error with the smaller number of variables was considered.

3) The validation trough dynamic prediction, which corresponds to the prediction of an arbitrary number of steps forward. In this case, the first point of the validation data group (initial condition) is used as an input to the network. In relation to the other points, only the information of the perturbation variable is used, as external information, and a feedback of the output variables is performed.

\section{B. Simulation results}

The neural network was trained by defining the $I_{a p p}$ current as an independent variable. $I_{a p p}$ is defined as a piecewise constant signal with 50 levels randomly defined with a uniform distribution. The value of the constant changes every 2000 integration steps (Figure 2).

To verify the robustness of the identification, parameters $g_{L}$ and $g_{K}$ were modified with a uniformly distributed noise $\eta(t)$ as follows:

$$
\begin{aligned}
g_{L} & =g_{L}(1+\eta(t)), \\
g_{K} & =g_{K}(1+\eta(t)) ;
\end{aligned}
$$

The noise amplitude value considered in this experiment is $8 \%$.

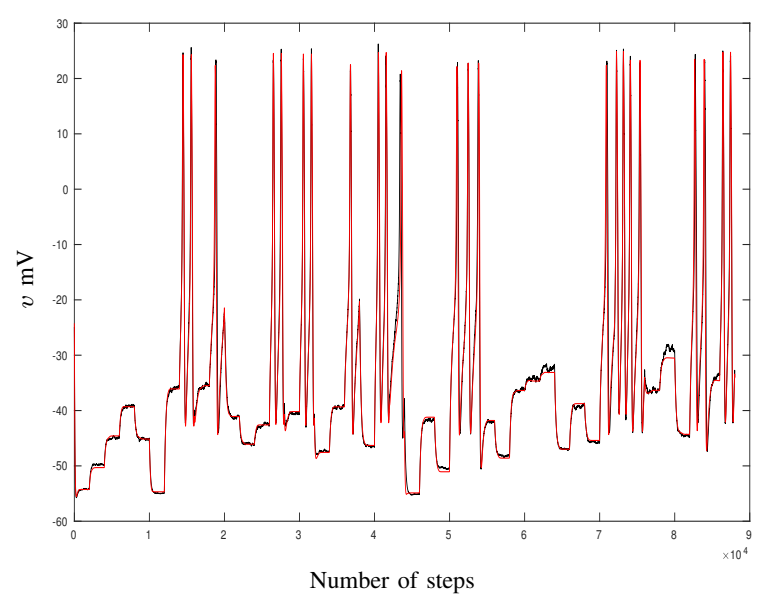

Fig. 3. Membrane voltage

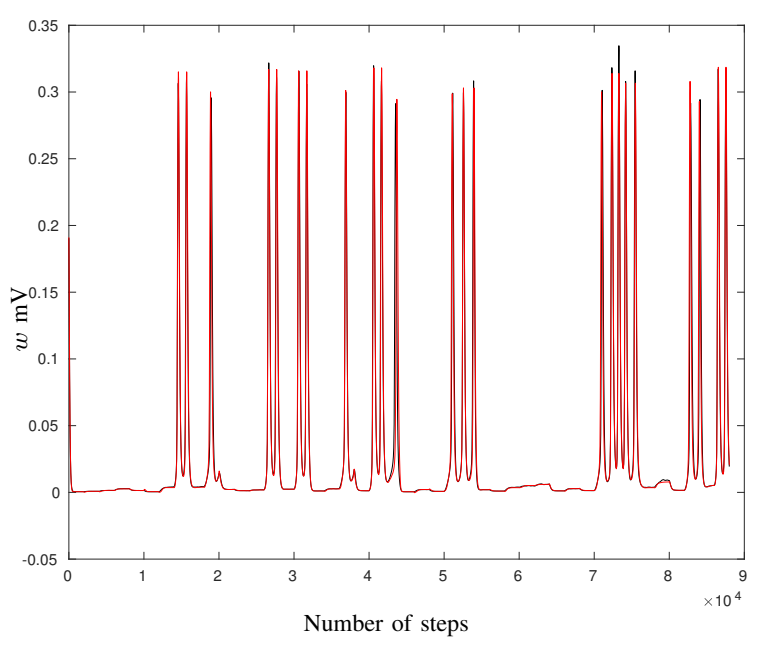

Fig. 4. Probability of the $K^{+}$ionic channel to be active

To solve the differential equations of the Morris-Lecar model, the Euler method was used with step $T_{s}=0.05 \mathrm{~ms}$ compatible with the sampling period of experimental data acquisition systems.

The solutions of the differential equations the model and the neural network prediction are depicted (actually overlapped) in the next figures. Figure 3 shows the results corresponding to variable $v$ and Figure 4 shows the results corresponding to variable $w$. As it can be verified from the results presented, the prediction in both subthreshold and trigger conditions is satisfactory. In Figure 5, the simulated and predicted variables are shown simultaneously in a phase-plane representation. As it can be appreciated the network is able to track, with high accuracy, the oscillations elicited when $I_{a p p}$ visits the parameter region with stable limit cycles, that is, when $I_{a p p}>I_{b i f}$. 


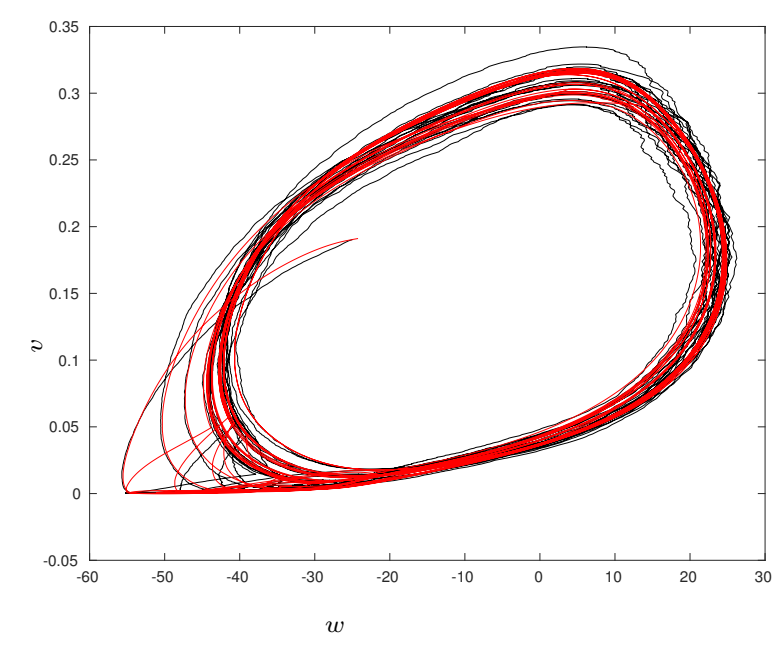

Fig. 5. $(v, w)$ Phase-portrait

\section{CONCLUSIONS AND FUTURE WORK}

We have tested the performance of a modified wavenet to identify parameters of a neuron model from its voltage traces. We show that, after training our artificial network with biologically plausible input currents, the network is able to identify the neuron's behaviour with high accuracy, thus obtaining a black box that can be further used for predictive goals. Interestingly, the interval of input currents used to train the network includes both current levels for which the neuron is quiescent and current levels that elicit spikes, which shows the ability of our network to identify abrupt changes in the bifurcation diagram, from almost linear input-output relationships to highly nonlinear ones. In this work, the simulations have been performed on a benchmark representative model, with the aim of providing a proof of concept, but our procedure can be easily extended to investigate the identification of other neuron models encoding more sophisticated types of dynamics (bursting, adaptation, mixed-mode oscillations, etc).

Even more importantly, our approach opens promising avenues when applied to real neurons, since it naturally leads to a heuristic model of a neuron just stimulating it in closedloop experiments (that is, using the dynamic-clamp, a wellknown electrophysiology technique) and using the output data to train the wavenet. In addition, this procedure would allow to associate a heuristic model to available data in neuroscience that has not gone through a careful channel dissection to biophysically characterize the corresponding neuron, even if the data does not come from electrophysiological recordings of a single cell; for instance, it could also provide heuristic models for populations of neurons. Finally, another application of our approach is to infer, via inverse control methods, the time course of the input current received by a neuron, which essentially corresponds to the synaptic input and thus provides a valuable information about neuron's connectivity, a paramount problem in neuroscience.

\section{ACKNOWLEDGMENT}

The authors would like to thank the Spanish grant MINECO-FEDER-UE MTM-2015-71509-C2-2-R (AG) and the Catalan Grant 2017SGR1049 (AG) 2017SGR0872 (EF). This work has been done in a long-term stay of Prof. Roqueiro at the UPC.

\section{REFERENCES}

[1] C. Morris and H. Lecar, Voltage Oscillations in the barnacle giant muscle fiber, Biophys J. 35, 193-213, 1981.

[2] A. L. Hodgkin and A. F. Huxley, The components of membrane conductance in the giant axon of Loligo, J Physiol. 116, 473-496, 1952.

[3] E. Izhikevich, Dynamical systems in neuroscience: the geometry of excitability and bursting, MIT Press, 2006.

[4] J. R. Rinzel and G. B. Ermentrout, Analysis of neural excitability and oscillations, in Methods in Neural Modeling, C. Koch and I. Segev Eds., 135-169, MIT Press, 1998.

[5] C. Ekanadham, D. Tranchina, and E.P. Simoncelli. A unified framework and method for automatic neural spike identification, Journal of Neuroscience Methods, 222, 47-55, 2014.

[6] D.E. Rumelhart, J.L. McClelland. Parallel Distributed Procesing: Explorations in the Microstrucuture of Cognition. MIT Press, 1987

[7] S. Haikin. Neural Networks - A Comprehensive Foundation. PrenticeHall, 1999

[8] S.A. Mallat. A Theory for Multiresolution Signal Decomposition: The Wavelet Representation, IEEE Trans. Pat. Anal Mach. Intel., 11(7), 674693, 1989.

[9] I. Daubechies. Ten Lectures on Wavelets, SIAM, 1992.

[10] G. Strang, T. Nguyen. Wavelets and Filter Banks Wellesley-Cambridge Press, 1996

[11] B.R. Bakshi, G. Stephanopoulos. Wave-Net: a Multiresolution, Hierarchical Neural Network with Localizad Learning, AIChE J., 39(1), 57-81.

[12] Q. Zhang, A.Benveniste. Wavelet Networks, IEEE Trans. Neural Networks, 3(6), 889-898.

[13] C.A. Claumann. Modelagem e controle de processos não lineares: Uma Aplicação de Algoritmos Genéticos no Treinamento de Redes Neurais Recorrentes. Dissertação de Mestrado, UFSC, Brasil.

[14] M. Pottmann, D.E. Seborg Identification of Nonlinear Process Using Reciprocal Multiquadratic Functions J. Process Control, 21, 956-980.

[15] M.A. Henson, D.E. Seborg An Internal Model Control Strategy for Nonlinear Systems, AIChE J., 37(7), 1991.

[16] N. Roqueiro. Redes de Wavelets na Modelagem de Processos não Lineares. Tese de Doutorado - COPPE/UFRJ, 1995

[17] C.A. Claumann, Desenvolvimento e aplicações de redes neurais wavelets $e$ da teoria de regularização na modelagem de processos. $\mathrm{PhD}$ Thesis, UFSC, Florianopolis, Brasil, 2003 